\title{
Abnormal expression of seven myogenesis-related genes in extraocular muscles of patients with concomitant strabismus
}

\author{
YUJUAN ZHU* , DAMING DENG ${ }^{*}$, CHONGDE LONG*, GUORONG JIN, \\ QINGJIONG ZHANG and HUANGXUAN SHEN
}

State Key Laboratory of Ophthalmology, Zhongshan Ophthalmic Center, Sun Yat-Sen University, Guangzhou, P.R. China

Received June 14, 2012; Accepted October 3, 2012

DOI: $10.3892 / \mathrm{mmr} .2012 .1149$

\begin{abstract}
Hyperplasia or hypoplasia of muscles gradually leads to strabismus. Myogenesis-related genes are involved in extraocular muscle development, including myogenic differentiation 1 (MYOD1), myogenin (MYOG), retinoblastoma 1 (RB1), cyclin-dependent kinase inhibitor 1A (P21), cyclin-dependent kinase inhibitor 1C (P57), insulin-like growth factor 1 (IGF1) and muscle creatine kinase (MCK). This study evaluated the expression of the above seven myogenesis-related genes by realtime quantitative RT-PCR in 18 resected extrocular muscles of patients with concomitant strabismus and 12 normal control muscle samples from one presumably healthy male $6 \mathrm{~h}$ after sudden mortality. We found that although there was a great divergence among the expression levels of 6 myogenesis-related regulatory factors, the relative expression patterns were similar in all the normal muscles, including the synergistic, antagonistic and yoke muscles. However, their expression levels in the 18 diseased extraocular muscles were abnormal; the expression levels of all the genes, with the exception of P57, were reduced in most of the diseased muscle tissues. These results imply that the abnormal expression of these myogenesis-related genes may contribute to concomitant strabismus.
\end{abstract}

\section{Introduction}

There are two main forms of strabismus, concomitant strabismus and incomitant strabismus. Concomitant strabismus is

Correspondence to: Dr Huangxuan Shen, Ophthalmic Genetics and Molecular Biology, Zhongshan Ophthalmic Center, Sun Yat-Sen University, 54 Xianlie Road, Guangzhou 510060, P.R. China

E-mail: shenhx@mail.sysu.edu.cn; zocshen@yahoo.com

*Contributed equally

Abbreviations: MR, medial rectus; LR, lateral rectus; SR, superior rectus; IR, inferior rectus; $\mathrm{SO}$, superior oblique; IO, inferior oblique; $\mathrm{XT}$, concomitant exotropia; ET, concomitant esotropia; NC, normal control

Key words: concomitant strabismus, myogenesis-related related genes, real-time RT-PCR characterized by an angle of deviation that remains the same in all directions of gaze, whichever eye is fixing. Strabismus is one of the most common problems in pediatric ophthalmology, affecting 3-5\% of the worldwide population $(1,2), 2-4 \%$ of the white population and $0.6 \%$ of Asians and Africans (3-5).

The pathogenesis of strabismus, particularly concomitant strabismus, remains poorly understood. It is believed that neural abnormalities and genetic and environmental factors are responsible for the occurrence of strabismus (6-8). Clinical observations have shown that the hypergenesis or hypoplasia of muscles may cause ocular muscles to become unbalanced; this may reflect a change in the neural input to an anatomically normal muscle and lead to strabismus, for example, the correlation between the overacted inferior oblique muscles and incomitant strabismus (9-12). Anatomic anomalies exist in $90 \%$ of the strabismus cases that occur before the patient is 6 years old. Furthermore, our previous study confirmed the abnormal expression of structural proteins in some of the 324 extraocular muscles of 278 patients with strabismus, including concomitant strabismus (unpublished data). Therefore, we aimed to acquire more information concerning the correlation between muscle development-related genes and concomitant strabismus.

Myogenic differentiation 1 (MYOD1), myogenin (MYOG), retinoblastoma 1 (RB1), cyclin-dependent kinase (CDK) inhibitor 1A (P21), CDK inhibitor 1C (P57) and insulin-like growth factor 1 (IGF1) are the key regulatory genes involved in the initiation and regulation of myogenesis (http://www. ans.iastate.edu/research/reecy/reecyfocus.html). Muscle creatine kinase $(\mathrm{MCK})$ is a marker of the late stage of muscle differentiation (13).

MYOD1 and MYOG are two key myogenic factors. MYOD1, which is only expressed in skeletal muscle and its precursors (14), regulates muscle cell differentiation by regulating the cell cycle and is a prerequisite for myogenic initiation. MYOG encodes a specific transcription factor for inducing myogenesis, which acts at a late stage of myogenesis and is essential for the formation of functional skeletal muscle $(15,16)$.

The cell cycle regulatory factors participate in myogenesis by interacting with myogenic factors. The RB1 protein, $\mathrm{pRb}$, is able to strengthen the activity of MYOD and MYOG during skeletal myogenesis as a transcriptional co-factor of MyoD (13,17-19). Furthermore, pRb controls entry into the late stages of the muscle differentiation (13). p21 is essential for the 
Table I. Clinical information of the individuals.

\begin{tabular}{rlrrll}
\hline Patient & Gender & Age (year) & Deviation & Eye & Muscle \\
\hline 1 & Female & 14 & ET & Right & LR \\
2 & Male & 10 & ET & Right & LR \\
3 & Male & 11 & ET & Left & LR \\
4 & Male & 15 & ET & Right & LR \\
5 & Male & 9 & ET & Right & LR \\
6 & Male & 20 & ET & Right & LR \\
7 & Male & 7 & ET & Right & LR \\
8 & Female & 18 & ET & Left & LR \\
9 & Female & 15 & ET & Right & MR \\
10 & Female & 16 & XT & Left & MR \\
11 & Female & 36 & XT & Right & MR \\
12 & Male & 16 & XT & Left & MR \\
13 & Male & 22 & XT & Left & MR \\
14 & Male & 10 & XT & Left & MR \\
15 & Male & 7 & XT & Right & MR \\
16 & Male & 6 & XT & Right & MR \\
17 & Female & 8 & XT & Left & MR \\
18 & Female & 18 & XT & Left & MR \\
\hline
\end{tabular}

ET, concomitant esotropia; XT, concomitant exotropia; LR, lateral rectus; $M R$, medial rectus.

cell cycle and differentiation in the adult myogenic progenitor cell (MPC) population (20). p57 is able to stabilize MYOD by direct binding (21-23). They are all induced by MYOD and play important roles in the cell cycle of muscle terminal differentiation $(24,25)$. IGF1 is able to promote skeletal muscle differentiation and repair muscle damage (26-28).

In order to illuminate the correlation between myogenesis and concomitant strabismus, we analyzed these 7 genes in 18 extraocular muscles of 18 patients with concomitant strabismus and 12 normal control muscles from one patient. The results demonstrated abnormal expression patterns of these 7 genes in the majority of the diseased extraocular muscles.

\section{Materials and methods}

Samples. The resected extraocular muscles were obtained from patients with strabismus during resection surgery at the Zhongshan Ophthalmic Center, Guangzhou, China. A total of 18 extraocular muscles from concomitant strabismus patients were analyzed in this study, including 10 medial rectus (MR) muscle tissues from 10 patients with concomitant exotropia (XT) and 8 lateral rectus (LR) muscle tissues from 8 patients with concomitant esotropia (ET). Some clinical information of the individuals are showed in Table I. Twelve normal control samples, including binocular MR, LR, superior rectus (SR), inferior rectus (IR), superior oblique (SO) and inferior oblique (IO) muscle samples, were obtained from one presumably healthy male $6 \mathrm{~h}$ after sudden mortality. The samples were immediately frozen in liquid nitrogen and stored at $-80^{\circ} \mathrm{C}$. Informed consent adhering to the tenets of the Declaration of Helsinki was obtained from all participants or their guardians
Table II. Primers for real-time PCR.

\begin{tabular}{llc}
\hline Gene name & \multicolumn{1}{c}{ Primer sequences $\left(5^{\prime} \rightarrow 3^{\prime}\right)$} & Tm $\left({ }^{\circ} \mathrm{C}\right)$ \\
\hline MYOD1 & F-CGCTCCAACTGCTCCGACGG & 66 \\
& R-GACACCGCCGCACTCTTCCC & \\
MYOG & F-GAGCTCACCCTGAGCCCCGA & 66 \\
& R-GCAGGCACTGGCCTGGACAG & \\
RB1 & F-AGCTGTGGGACAGGGTTGTGTC & 64 \\
& R-CAACCTCAAGAGCGCACGCC & \\
MCK & F-GCCACGGGGGCTACAAACCC & 66 \\
& R-GTGGGGGCAACGTGTAGCCC & \\
P21 & F-GCTGTCCCCTGCAGCAGAGC & 65 \\
& R-TGCATCCAGGAGGCCCGTGA & \\
P57 & F-CTCTTGCGCGGGGTCTGCTC & 63 \\
& R-AACGGCGCGGCGATCAAGAA & \\
IGF1 & F-GGGCGCCTCAGACAGGCATC & 65 \\
& R-CAGGCTTGAGGGGTGCGCAA & \\
GAPDH & F-CCCGCTTCGCTCTCTGCTCC & 65 \\
\multirow{2}{*}{-actin } & R-ACCAGGCGCCAATACGACC & \\
& F-CGAGCACAGAGCCTCGCCTTTGC & 66 \\
& R-ACATGCCGGAGCCGTTGTCGAC & \\
\hline
\end{tabular}

Tm, temperature; MYOD1, myogenic differentiation 1; MYOG, myogenin; RB1, retinoblastoma 1; MCK, muscle creatine kinase; IGF1, insulin-like growth factor 1 .

before the study. This study was approved by the Institutional Review Board of Zhongshan Ophthalmic Center.

Real-time quantitative RT-PCR. Total RNA was isolated using a commercial reagent TRIzol (Invitrogen Life Technologies, Carlsbad, CA, USA) and $1 \mu \mathrm{g}$ RNA was reverse transcribed to cDNA using a commercial reagent kit (PrimeScript ${ }^{\circledR}$ RT reagent kit; Takara Bio, Inc., Shiga, Japan). The cDNA (1 $\mu \mathrm{l})$ was diluted (1:10) for real-time PCR. Real-time PCR was performed using a reagent kit $\left(\mathrm{SYBR}^{\circledR}\right.$ Premix Ex Taq ${ }^{\mathrm{TM}}$, Takara Bio, Inc.) following the manufacturer's instructions for the ABI Prism 7000 sequence detection system (Applied Biosystems, Foster City, CA, USA). The primers used for real-time PCR are shown in Table II. GAPDH were used as an internal control to normalize the gene expression levels. Relative transcript abundance was quantified by the $2^{-\Delta \Delta C T}$ method. Data represent the mean of triplicate measurements and are reported as the mean fold change ( $x$-fold) + SD.

\section{Results}

Expression patterns of 6 myogenesis regulatory factors are similar in the normal extraocular muscles. In this study, we first examined the expression of 6 myogenesis regulatory factors in normal samples, i.e., all 12 extraocular muscles from the normal eye, including bilateral MR, LR, SR, IR, SO and IO muscles (Fig 1). We found a similar expression pattern in all the muscles, including the synergistic, antagonistic and yoke muscles. In the synergistic muscles, including MR-SR-IR 

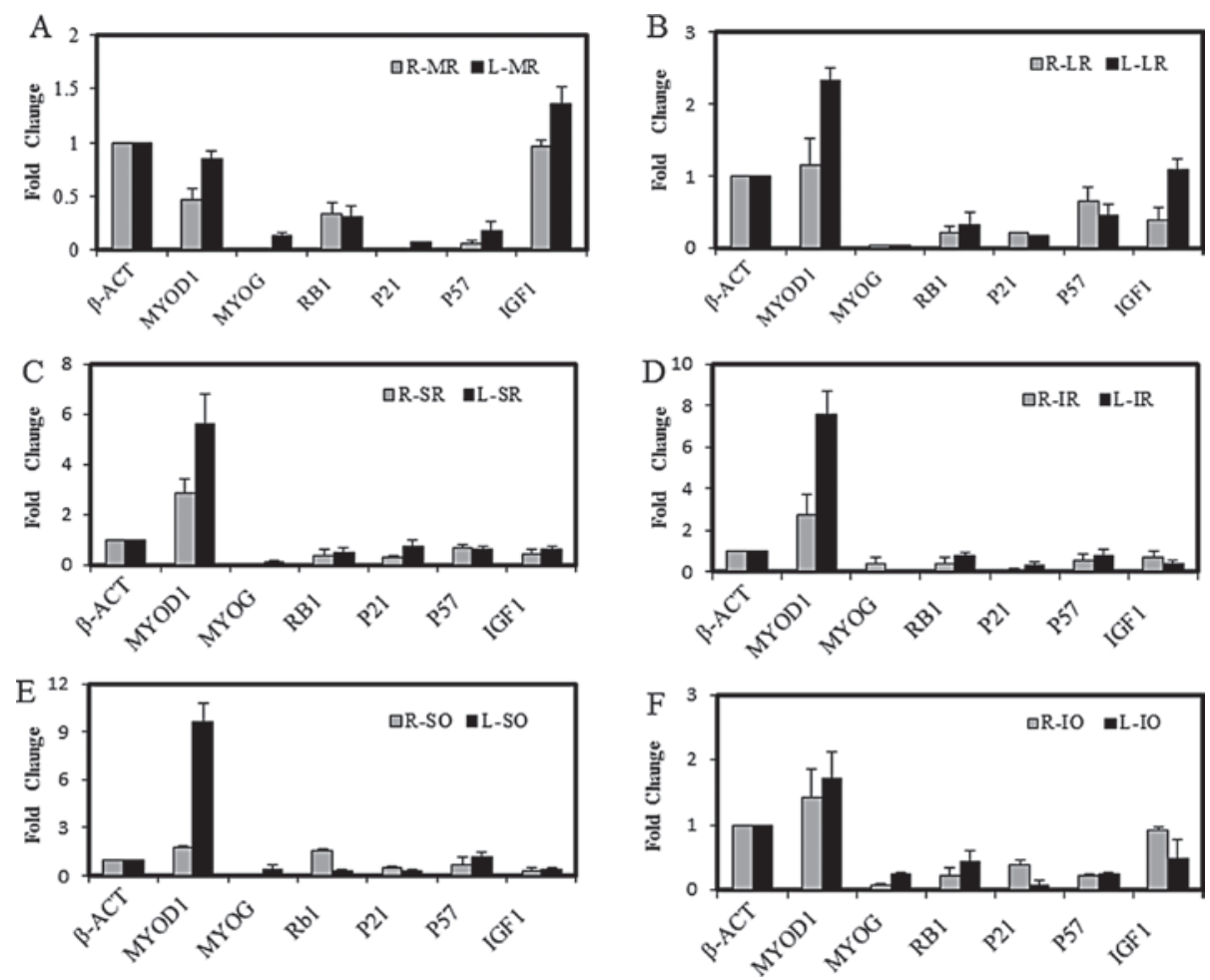

Figure 1. Expression of 6 myogenesis regulatory genes in all 12 normal extraocular muscles. The relative level of all the six genes: MYOD1, myogenic differentiation 1; MYOG, myogenin; RB1, retinoblastoma 1; MCK, muscle creatine kinase; IGF1, insulin-like growth factor 1 in all 6 extraocular muscles of same eye ball were analyzed: (A) MR, medial rectus; (B) LR, lateral rectus; (C) SR, superior rectus; (D) IR, inferior rectus; (E) SO, superior oblique; and (F) IO, inferior oblique. The total RNA was from all 12 extraocular muscles from the right eye (R) and the left eye (L) of the same control. Using the specific primers, the expression levels of 6 factors and $\beta$-actin were quantified by real-time quantitative RT-PCR. $\beta$-ACT, $\beta$-actin.

(Fig. 1A, C and D) and LR-SO-IO (Fig. 1B, E and F), MYOD1 levels were invariably higher and MYOG levels were lower than those of other genes. For the other factors, the levels of IGF1 were close to those of $\beta$-actin in most samples. The expression levels of the cell cycle regulatory factors RB1, P21 and P57 were low in the MR, LR and IO muscles but comparable to those of $\beta$-actin in the other 3 types of muscles. The expression pattern was similar in the antagonistic muscles and the yoke muscles (Fig. 1). Although there was a divergence in expression levels among the 6 myogenesis-related regulatory factors, there was a similar expression pattern in all the 12 normal binocular extraocular muscles.

Abnormal expression of 7 myogenesis-related genes in 18 extraocular muscles of patients with concomitant strabismus. Generally, we obtained the resectional MR muscle from the XT patients and LR muscle from the ET patients by surgery. Therefore, we detected the expression of the 7 myogenesis-related genes in XT-MR and ET-LR muscles in this study. Fig. 2 shows the abnormal expression of all 7 factors in the diseased muscles.

The expression levels of MYOD1 and MYOG were markedly reduced in almost all of the samples (Fig. 2A). The expression levels of MYOD1 were reduced significantly in 7 of the $8 \mathrm{LR}$ and 8 of the 10 MR samples, and those of MYOG decreased in 7 of the $8 \mathrm{LR}$ and all $10 \mathrm{MR}$ samples. Although the levels of MYOD1 and MYOG were downregulated, the relative expression pattern between them was similar to that in normal tissues: the levels of MYOD1 were clearly higher than those of MYOG.
The expression of the three cell cycle regulators was different in diseased tissues (Fig. 2B). The expression levels of RB1 were reduced in 5 of the $8 \mathrm{LR}$ and 7 of the $10 \mathrm{MR}$ samples but increased in the other samples (Fig. 2B-a and b). The expression levels of P21 were decreased in all 18 samples, particularly in the MR tissue, but the expression levels of P57 were increased in 7 of the $8 \mathrm{LR}$ and 7 of the $10 \mathrm{MR}$ samples (Fig. 2B-c-f).

The expression levels of the other two factors, IGF1 and MCK, were reduced in most of the samples compared with their levels in the normal controls (Fig. 2C). The IGF1 levels were reduced in 6 of the $8 \mathrm{LR}$ and most of the $10 \mathrm{MR}$ tissues. MCK levels were decreased in 6 of the 8 LR and 8 of the 10 MR tissues, but increased in the others.

These results reveal the abnormal expression of these myogenesis genes in the extraocular muscles of patients with concomitant strabismus.

\section{Discussion}

An obstacle to the understanding of eye muscle pathology is the lack of a practical way to obtain tissue samples of the entire muscle from normal individuals and patients with strabismus (29). In this study, we examined the mRNA levels of 7 myogenesis-related genes in 18 extraocular muscles from 18 patients with concomitant strabismus and 12 normal control samples from one presumably healthy individual. We found that a similar expression pattern of these genes was presented in all the normal tissues. However, their expression was 
A
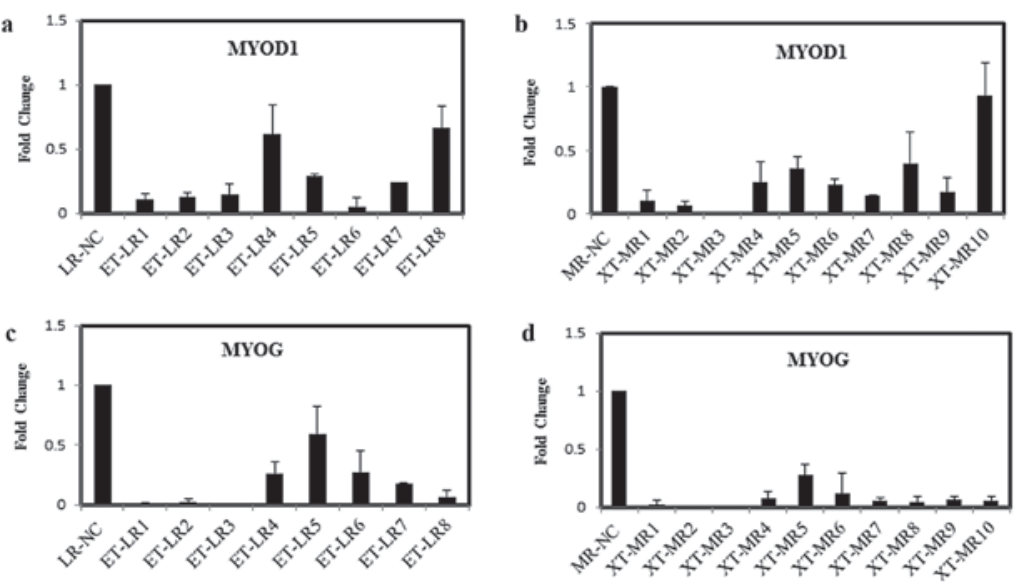

B
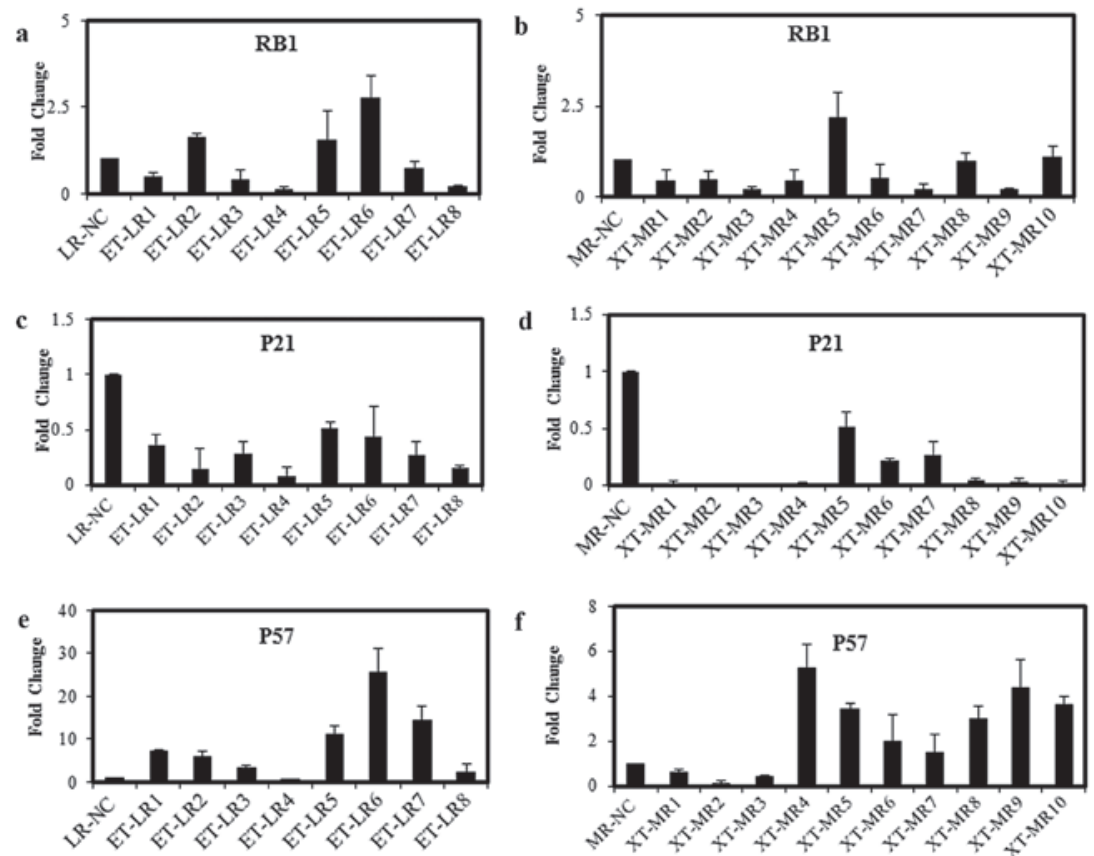

C
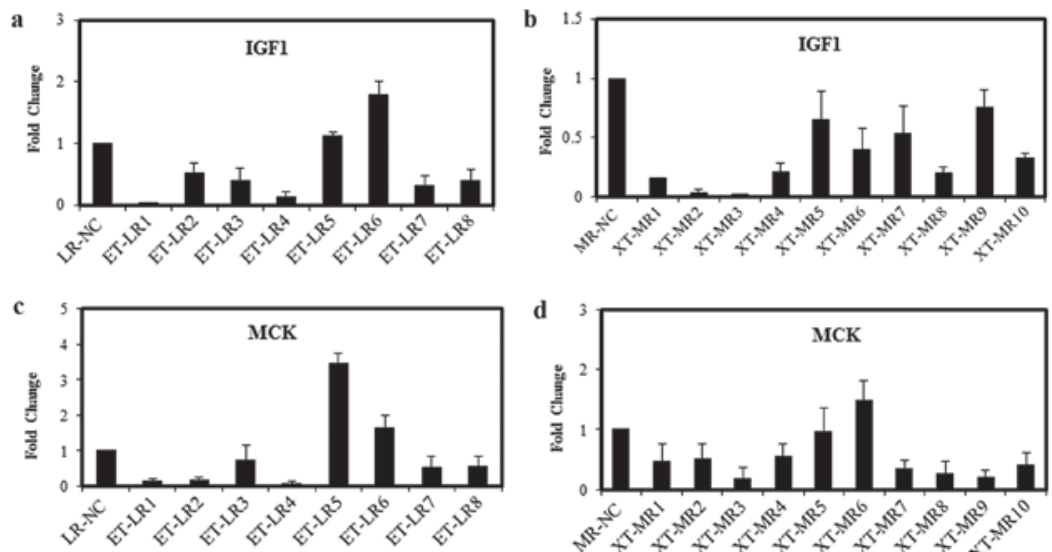

Figure 2. Expression of 7 myogenesis related genes in the 18 diseased concomitant exotropia-medial rectus (XT-MR) and concomitant esotropia-lateral rectus (ET-LR) tissues. (Aa and b) The level of MYOD1 in diseased ET-LR and XT-MR relative to normal. (Ac and d) The level of MYOG. (Ba and b) The level of RB1 in diseased ET-LR and XT-MR relative to the normal. ( $\mathrm{Bc}$ and d) The level of P21. (Be and f) The level of P57. (Ca and b) The level of IGF1 in diseased ET-LR and XT-MR relative to the normal. (Cc and d) The level of MCK. NC, normal control; MYOD1, myogenic differentiation 1; MYOG, myogenin; RB1, retinoblastoma 1; MCK, muscle creatine kinase; IGF1, insulin-like growth factor 1. 
abnormal in the extraocular muscles of patients with concomitant strabismus.

Together with several other genes related to myogenesis, myogenic factors during muscle regeneration, including MYOD, myogenic factor (Myf)5, myogenin and Myf4, are essential for skeletal muscle determination and the differentiation of skeletal muscle tissue. MYOD knockout mice are deficient in skeletal muscle regeneration (30-32). In activated skeletal satellite cells, differentiating signals upregulate MYOD and activate the transcription of MYOD target genes, result in myogenic differentiation (33). In the current study, we found that the level of MYOD1 was always higher than that of MYOG in normal tissues and also in the diseased tissues. Therefore, we suspected that this expression pattern is required for normal muscle maturation. Moreover, this pattern was also present in all the synergistic and antagonistic muscles, as well as in the yoke muscles. This may maintain a balance among all the extraocular muscles and may be necessary for maintaining normal eye movement.

In the extraocular muscles of the concomitant strabismus patients, the expression levels of MYOD1 and MYOG were reduced by $70-80 \%$. Repressed MYOG expression is responsible for impaired myoblast differentiation (34). The repressed levels of MYOD1 and MYOG imply that the myogenesis process, including differentiation, fusion and growth, was impaired in the concomitant strabismus patients. This is likely to disrupt the formation of functional muscles and the development of extraocular muscles and, therefore, lead to the gradual development of concomitant strabismus. However, downregulation of myogenin may reactivate the cell cycle, probably through the downregulation of the endogenous expression of MYOD (35). Therefore, the repression of MYOD1 and MYOG levels may be a separate self-healing mechanism of the impaired muscles.

Other regulatory factors functioning in muscle differentiation, specifically RB1, P21, P57 and IGF1, constitute a regulatory network with myogenic factors that interact with each other directly or indirectly. pRb interacts with MYOD and MYOG, and controls entry into the later stages of the muscle differentiation process (13,36-38). The CDK inhibitors, P21 and P57, block the cell cycle by repressing the activity of CDKs. The regulatory factors, particularly the cell cycle inhibitors, are theoretically highly expressed in normal skeletal muscle. However, our data revealed that the levels of RB1, P21 and IGF1, particularly those of P21, were reduced in the majority of the diseased tissues with respect to their levels in the normal controls. The levels of another cell cycle inhibitor, P57, were increased in most of the diseased samples. Therefore, decreased expression of RB1 and P21 may be the reason for muscle overdevelopment and increased expression of P57 may be a compensation for decreased RB1, P21 and IGF1 levels. As MYOD is able to induce the expression of p57 in cells lacking p21 $(24,39)$, repressed P21 and increased P57 levels may be caused by the reduction of MYOD1 levels. Regardless of what caused the changes in the 3 genes, the balance of cell cycle regulation was disrupted in the differentiation and growth of the extraocular muscles in the patients with concomitant strabismus.

The regulatory network of myogenic factors and other muscle growth regulators maintains the normal development and maturation of skeletal muscle, including extraocular muscle. Our results confirmed the abnormality in the levels of these myogenesis-related genes, which may contribute to concomitant strabismus and augment the pathology of the extraocular muscle of the concomitant strabismus patients. Additional samples and candidate genes should be studied in the future. Further studies are required to identify the mechanism involving these genes and determine the overall correlation between the myogenic process and the occurrence of strabismus.

\section{Acknowledgements}

The authors thank all patients for their participation. This study was supported by grants from the National Natural Science Foundation of China (81170891).

\section{References}

1. Ziakas NG, Woodruff G, Smith LK and Thompson JR: A study of heredity as a risk factor in strabismus. Eye (London) 16: 519-521, 2002.

2. Arora A, Williams B, Arora AK, McNamara R, Yates J and Fielder A: Decreasing strabismus surgery. Br J Ophthalmol 89: 409-412, 2005;

3. Hu DN: Prevalence and mode of inheritance of major genetic eye diseases in China. J Med Genet 24: 584-588, 1987.

4. Robaei D, Rose KA, Kifley A, Cosstick M, Ip JM and Mitchell P: Factors associated with childhood strabismus: findings from a population-based study. Ophthalmology 113: 1146-1153, 2006.

5. Abrahamsson M, Magnusson G and Sjöstrand J: Inheritance of strabismus and the gain of using heredity to determine populations at risk of developing strabismus. Acta Ophthalmol Scand 77: 653-657, 1999.

6. Mustari MJ and Ono S: Neural mechanisms for smooth pursuit in strabismus. Ann N Y Acad Sci 1233: 187-193, 2011.

7. Wilmer JB, Backus BT. Genetic and environmental contributions to strabismus and phoria: evidence from twins. Vision Res 49: 2485-2493, 2009.

8. Michaelides M and Moore AT: The genetics of strabismus. J Med Genet 41: 641-646, 2004.

9. Kushner BJ: Incomitant strabismus: does extraocular muscle form denote function? Arch Ophthalmol 128: 1604-1609, 2010.

10. Chang YH, Ma KT, Lee JB and Han SH: Anterior transposition of inferior oblique muscle for treatment of unilateral superior oblique muscle palsy with inferior oblique muscle overaction. Yonsei Med J 45: 609-614, 2004.

11. Yu X, Mai G, Yu H, Deng D, Lin X, Chen J and Wu H: Study on ocular torsion of V patterns strabismus. Yan Ke Xue Bao 19: 160-164, 2003.

12. Mellott ML, Scott WE, Ganser GL and Keech RV: Marginal myotomy of the minimally overacting inferior oblique muscle in asymmetric bilateral superior oblique palsies. J AAPOS 6: 216-220, 2002.

13. Novitch BG, Spicer DB, Kim PS, Cheung WL and Lassar AB: $\mathrm{pRb}$ is required for MEF2-dependent gene expression as well as cell-cycle arrest during skeletal muscle differentiation. Curr Biol 9: 449-459, 1999.

14. Weintraub H, Davis R, Tapscott S, et al: The myoD gene family: nodal point during specification of the muscle cell lineage. Science 251: 761-766, 1991.

15. Hasty KA, Wu H, Byrne M, et al: Susceptibility of type I collagen containing mutated alpha 1(1) chains to cleavage by human neutrophil collagenase. Matrix 13: 181-186, 1993.

16. Apponi LH, Corbett AH and Pavlath GK: RNA-binding proteins and gene regulation in myogenesis. Trends Pharmacol Sci 32: 652-658, 2011.

17. Gu W, Schneider JW, Condorelli G, Kaushal S, Mahdavi V and Nadal-Ginard B: Interaction of myogenic factors and the retinoblastoma protein mediates muscle cell commitment and differentiation. Cell 72: 309-324,1993

18. Skapek SX, Pan YR and Lee EY: Regulation of cell lineage specification by the retinoblastoma tumor suppressor. Oncogene 25: 5268-5276, 2006. 
19. Thomas DM, Carty SA, Piscopo DM, Lee JS, Wang WF, Forrester WC and Hinds PW: The retinoblastoma protein acts as a transcriptional coactivator required for osteogenic differentiation. Mol Cell 8: 303-316, 2001

20. Hawke TJ, Meeson AP, Jiang N, Graham S, Hutcheson K, DiMaio JM and Garry DJ: p21 is essential for normal myogenic progenitor cell function in regenerating skeletal muscle. Am J Physiol Cell Physiol 285: C1019-C1027, 2003.

21. Park CW and Chung JH: Age-dependent changes of p57(Kip2) and $\mathrm{p} 21(\mathrm{Cip} 1 / \mathrm{Waf} 1)$ expression in skeletal muscle and lung of mice. Biochim Biophys Acta 1520: 163-168, 2001.

22. Reynaud EG, Leibovitch MP, Tintignac LA, Pelpel K, Guillier M and Leibovitch SA: Stabilization of MyoD by direct binding to p57(Kip2). J Biol Chem 275: 18767-18776, 2000.

23. Zhang P, Wong C, Liu D, Finegold M, Harper JW and Elledge SJ. p21(CIP1) and p57(KIP2) control muscle differentiation at the myogenin step. Genes Dev 13: 213-224, 1999.

24. Guo K, Wang J, Andrés V, Smith RC and Walsh K: MyoD-induced expression of p21 inhibits cyclin-dependent kinase activity upon myocyte terminal differentiation. Mol Cell Biol 15: 3823-3829, 1995.

25. Vaccarello G, Figliola R, Cramerotti S, Novelli F and Maione R p57Kip2 is induced by MyoD through a p73-dependent pathway. J Mol Biol 356: 578-588, 2006.

26. Kandalla PK, Goldspink G, Butler-Browne G and Mouly V: Mechano growth factor E peptide (MGF-E), derived from an isoform of IGF-1, activates human muscle progenitor cells and induces an increase in their fusion potential at different ages. Mech Ageing Dev 132: 154-162, 2011

27. Matheny RW Jr, Nindl BC and Adamo ML: Minireview: Mechano-growth factor: a putative product of IGF-I gene expression involved in tissue repair and regeneration. Endocrinology 151: 865-875, 2010.

28. Hill $\mathrm{M}$ and Goldspink G: Expression and splicing of the insulin-like growth factor gene in rodent muscle is associated with muscle satellite (stem) cell activation following local tissue damage. J Physiol 549: 409-418,2003.

29. Spencer RF and Porter JD: Biological organization of the extraocular muscles. Prog Brain Res 151: 43-80, 2006.
30. Cornelison DD, Olwin BB, Rudnicki MA and Wold BJ: MyoD(-/-) satellite cells in single-fiber culture are differentiation defective and MRF4 deficient. Dev Biol 224: 122-137, 2000.

31. White JD, Scaffidi A, Davies M, McGeachie J, Rudnicki MA and Grounds MD: Myotube formation is delayed but not prevented in MyoD-deficient skeletal muscle: studies in regenerating whole muscle grafts of adult mice. J Histochem Cytochem 48: $1531-1544,2000$

32. Schuierer MM, Mann CJ, Bildsoe H, Huxley C and Hughes SM: Analyses of the differentiation potential of satellite cells from myoD-/-, mdx, and PMP22 C22 mice. BMC Musculoskelet Disord 6: 15, 2005.

33. Mokalled MH, Johnson AN, Creemers EE and Olson EN: MASTR directs MyoD-dependent satellite cell differentiation during skeletal muscle regeneration. Genes Dev 26: 190-202, 2012.

34. Lin X, Yang X, Li Q, et al: Protein tyrosine phosphatase-like A regulates myoblast proliferation and differentiation through MyoG and the cell cycling signaling pathway. Mol Cell Biol 32: 297-308, 2011.

35. Mastroyiannopoulos NP, Nicolaou P, Anayasa M, Uney JB and Phylactou LA: Down-regulation of myogenin can reverse terminal muscle cell differentiation. Plos One 7: e29896, 2012.

36. Burkhart DL and Sage J: Cellular mechanisms of tumour suppression by the retinoblastoma gene. Nat Rev Cancer 8: 671-682, 2008

37. Chen TT and Wang JY: Establishment of irreversible growth arrest in myogenic differentiation requires the RB LXCXE-binding function. Mol Cell Biol 20: 5571-5580, 2000.

38. Sellers WR, Novitch BG, Miyake S, et al: Stable binding to $\mathrm{E} 2 \mathrm{~F}$ is not required for the retinoblastoma protein to activate transcription, promote differentiation, and suppress tumor cell growth. Genes Dev 12: 95-106, 1998.

39. Figliola R and Maione R: MyoD induces the expression of p57Kip2 in cells lacking p21Cip1/Waf1: overlapping and distinct functions of the two cdk inhibitors. J Cell Physiol 200: 468-475, 2004. 